This is an electronic reprint of the original article. This reprint may differ from the original in pagination and typographic detail.

Author(s): Föhr, Tiina; Tolvanen, Asko; Myllymäki, Tero; Järvelä-Reijonen, E.; Peuhkuri, K.;

Rantala, S.; Kolehmainen, M.; Korpela, R.; Lappalainen, Raimo; Ermes, M.; Puttonen, S.; Rusko, Heikki; Kujala, Urho

Title: $\quad$ Physical activity, heart rate variability-based stress and recovery, and subjective stress during a 9-month study period

Year: $\quad 2017$

Version:

Please cite the original version:

Föhr, T., Tolvanen, A., Myllymäki, T., Järvelä-Reijonen, E., Peuhkuri, K., Rantala, S., Kolehmainen, M., Korpela, R., Lappalainen, R., Ermes, M., Puttonen, S., Rusko, H., \& Kujala, U. (2017). Physical activity, heart rate variability-based stress and recovery, and subjective stress during a 9-month study period. Scandinavian Journal of Medicine and Science in Sports, 27(6), 612-621. https://doi.org/10.1111/sms.12683

All material supplied via JYX is protected by copyright and other intellectual property rights, and duplication or sale of all or part of any of the repository collections is not permitted, except that material may be duplicated by you for your research use or educational purposes in electronic or print form. You must obtain permission for any other use. Electronic or print copies may not be offered, whether for sale or otherwise to anyone who is not an authorised user. 


\title{
Physical activity, heart rate variability-based stress and recovery, and subjective stress during a 9-month study period
}

\author{
T. Föhr ${ }^{1}$, A. Tolvanen ${ }^{2}$, T. Myllymäki ${ }^{3}$, E. Järvelä-Reijonen ${ }^{4}$, K. Peuhkuri ${ }^{5}$, S. Rantala ${ }^{5}$, M. Kolehmainen ${ }^{4}$, \\ R. Korpela ${ }^{5}$, R. Lappalainen ${ }^{3}$, M. Ermes ${ }^{6}$, S. Puttonen ${ }^{7,8}$, H. Rusko ${ }^{9}$, U.M. Kujala ${ }^{1}$ \\ ${ }^{1}$ Department of Health Sciences, University of Jyväskylä, Jyväskylä, Finland, ${ }^{2}$ Methodology Centre for Human Sciences, \\ University of Jyväskylä, Jyväskylä, Finland, ${ }^{3}$ Department of Psychology, University of Jyväskylä, Jyväskylä, Finland, ${ }^{4}$ Institute of \\ Public Health and Clinical Nutrition, Clinical Nutrition, University of Eastern Finland, Kuopio, Finland, ${ }^{5}$ Medical Faculty, \\ Pharmacology, Medical Nutrition Physiology, University of Helsinki, Helsinki, Finland, ${ }^{6}$ VTT Technical Research Centre of \\ Finland, Tampere, Finland, ${ }^{7}$ Finnish Institute of Occupational Health, Helsinki, Finland, ${ }^{8}$ Institute of Behavioral Sciences, \\ University of Helsinki, Helsinki, Finland, ${ }^{9}$ Department of Biology of Physical Activity, University of Jyväskylä, Jyväskylä, \\ Finland \\ Corresponding author: Tiina Föhr, University of Jyväskylä, P.O. Box 35, FIN-40014 Jyväskylä, Finland. Tel: + 358408054726 , \\ Fax: + 35814 617423,E-mail: tiina.fohr@jyu.fi
}

Accepted for publication 1 March 2016

\begin{abstract}
The aim of this study was to investigate the association between physical activity (PA) and objective heart rate variability (HRV)-based stress and recovery with subjective stress in a longitudinal setting. Working-age participants $(n=221 ; 185$ women, 36 men $)$ were overweight (body mass index, $25.3-40.1 \mathrm{~kg} / \mathrm{m}^{2}$ ) and psychologically distressed $(\geq 3 / 12$ points on the General Health Questionnaire). Objective stress and recovery were based on HRV recordings over 1-3 work days. Subjective stress was assessed with the Perceived Stress Scale and PA level with a questionnaire. Data were collected at three time points: baseline, 10 weeks post intervention, and at
\end{abstract}

the 36-week follow-up. We adopted a latent growth model to investigate the initial level and change in PA, objective stress and recovery, and subjective stress at the three measurement time points. The results showed that initial levels of PA $(P<0.001)$ and objective stress $(P=0.001)$ and recovery $(P<0.01)$ were associated with the change in subjective stress. The results persisted after adjustment for intervention group. The present results suggest that high PA and objectively assessed low stress and good recovery have positive effects on changes in subjective stress in the long-term.
Sedentary lifestyle and obesity are major concerns in today's society because they are risk factors for multiple adverse health outcomes. Longitudinal studies have shown the beneficial effects of physical activity (PA) on health (Physical Activity Guidelines Advisory Committee, 2008; Gerber \& Pühse, 2009). Furthermore, the evidence supports the significance of PA in the treatment of many chronic diseases (Pedersen \& Saltin, 2006; Kujala, 2009). Additionally, an increase in PA has been found to be associated with improved psychological well-being, including decreased job stress (Conn et al., 2009; Thøgersen-Ntoumani et al., 2015). However, individuals' wellness consists of both psychological and physiological well-being (Dodge et al., 2012). For instance, psychological stress often causes sympathetic responses in the autonomic nervous system (ANS), such as reduced heart rate variability (HRV) (Chandola et al., 2010). These physiological stress response reactions disappear during recovery; thus, successful recovery after a stressful situation is a central issue in stress management (Kinnunen, 2005).

Long-term monitoring of health in real-life settings is an important element of health or wellness management (Lappalainen et al., 2005; Verberk et al., 2007), and an HRV-based assessment of the physiological symptoms of stress is suggested to be a feasible method for this kind of long-term monitoring. HRV refers to the variation in intervals between consecutive heartbeats originating from the sinus node and reflects cardiac autonomic modulation non-invasively (Task Force, 1996). Thus, typically a healthy person is characterized by high HRV with efficient autonomic mechanisms and good adaptation ability, while reduced HRV is an indicator of an abnormal and insufficiently adapted ANS (Porges, 1992, 1995; Vanderlei et al., 2009). HRV is an independent predictor of mortality (Thayer et al., 2010). Furthermore, both subjective stress (Thayer et al., 2010; Jarczok et al., 2013) and obesity 


\section{Föhr et al.}

(Zahorska-Markiewicz et al., 1993) are found to be associated with decreased HRV.

The majority of HRV studies have used traditional time-domain and frequency-domain HRV variables such as root mean square of the successive differences. These time-domain and frequency-domain variables are very individual which limits their usability in stress assessment. However, it is also possible to provide applied heart rate (HR) and HRV-derived stress and recovery variables that include information that is difficult to obtain from traditional measures of HRV (Teisala et al., 2014). These novel variables consider factors such as HRV-derived respiratory variables and individual resting HR and HRV values. New objective approaches that consider the individuality of HRV could provide additional insight together with the subjective approaches often used in the assessment of stress and recovery.

Little is known about the association between objective physiological HRV-based stress and recovery with subjective psychological stress in a longitudinal setting. To the best of our knowledge, this study is unique in its focus on the association between PA and HRV-based stress and recovery with subjective stress in a longitudinal setting. The aim of the study was to investigate the effects of PA and objective stress and recovery on subjective stress among overweight and psychologically distressed working-age adults over a 9-month study period.

\section{Materials and methods \\ Participants}

The present longitudinal study included individuals $(n=221$; 185 women, 36 men) of different occupations with symptoms of metabolic syndrome and psychological distress who met the inclusion criteria in the initial screening for a controlled, randomized trial published previously (Lappalainen et al., 2014). Data collection for the study was done at three time points: baseline, 10 weeks post intervention, and at the 36week follow-up. The participants were randomly allocated into one of three low-intensity psychological intervention groups (internet-based, mobile-based, or face-to-face) or control group. The three intervention groups were described in detail previously (Lappalainen et al., 2014). The inclusion criteria included perceived psychological stress indicated by at least 3/12 points on the General Health Questionnaire (Makowska et al., 2002) and self-reported body mass index (BMI) between 27 and $34.9 \mathrm{~kg} / \mathrm{m}^{2}$. The participants did not have any severe chronic illnesses, and their current regular medication and changes in medication during the study period were reported. At the baseline, 79 participants had regular medication of those eight had a change in their medication and 13 participants started regular medication during the study period.

Available data on objective stress and recovery (HRV recording) and subjective stress (Perceived Stress Scale; PSS) were an additional inclusion criterion, and the use of HRVaffecting $\alpha$ - or $\beta$-adrenergic blocking agents was a further exclusion criterion for the analyses of this study. Finally, the subjects included in this study consisted of 221 individuals of whom $208(94 \%)$ participated in the 10 -week post-intervention measurements and $199(90 \%)$ in the 36-week follow-up measurements. The reasons some subjects did not complete the study were health reasons $(n=6)$, life situation/motivational reasons $(n=12)$, or unknown reasons $(n=4)$. The characteristics of the participants at baseline, 10 -week postintervention, and 36-week follow-up are presented in Table 1.

All participants were informed about the initial study and provided written informed consent prior to any measurements. The study was conducted according to the Declaration of Helsinki, and the study protocol was approved by the ethics committee of the Central Finland Health Care District.

\section{Measurements}

The measurements included an assessment of objective stress and recovery based on HRV and subjective stress based on PSS. Additionally, PA and body composition were assessed. All measurements were performed at baseline, 10-week postintervention, and 36-week follow-up.

Objective HRV-based stress and recovery were determined from the beat-to-beat $\mathrm{R}-\mathrm{R}$ interval recordings in real-life settings using a Firstbeat Bodyguard measurement device (Firstbeat Technologies Ltd, Jyväskylä, Finland) over 1-3 work days. The data were then analyzed using the Firstbeat Analysis Server software (version 5.3.0.4), which included a powerful artifact detection and correction feature for irregular ectopic beats, and signal noise. Second-by-second indices reflecting the activities of the sympathetic and parasympathetic nervous systems were calculated with the short-time Fourier transform method. The software categorizes the data into different physiological states, such as stress, recovery, and the PA of different intensities by taking into account individual characteristics (e.g., individual levels and scales of HR and HRV and the individual relationships between HRV and autonomic control) (Martinmäki et al., 2006). Stress is detected when sympathetic activity of the ANS dominates over parasympathetic activity without metabolic requirements caused by PA. Recovery is detected when parasympathetic activity dominates the ANS. In the categorization, second-bysecond HRV indices, HRV-derived respiration rate, oxygen consumption calculated by $\mathrm{HR}$, HRV-derived respiration rate, and on-off kinetics, and parameter describing excess-post exercise oxygen consumption are used with neural network data modeling (for more details, see the white papers by Firstbeat Technologies Ltd). The intensity of stress reaction is calculated from the HR, high-frequency $(0.15-0.4 \mathrm{~Hz})$, and low-frequency $(0.04-0.15 \mathrm{~Hz})$ components of HRV and respiratory variables. The intensity of stress is high when HR is elevated, HRV is reduced, and the frequency distribution of HRV is inconsistent because of changes in the respiratory period. The intensity of recovery is calculated from the HR and high-frequency component of HRV, and it is high when HR is low and the high-frequency component of HRV is high and regular. Thereafter, the software creates variables, such as stress index and recovery index, to describe the state of the body (Saalasti, 2003; Kettunen \& Saalasti, 2005; Kettunen \& Saalasti, 2008; Firstbeat Technologies Ltd, 2014). The stress index, which characterizes the magnitude of stress processes during the whole day, was used as an indicator of objective stress. The recovery index, which characterizes the magnitude of recovery processes during sleep (the 4-h window for determining the recovery index is set to start $30 \mathrm{~min}$ after going to bed), was used as an indicator of objective recovery in this study. These indices describe the mean intensity of the recognized stress/recovery reactions (theoretically ranging from 0 to $\infty$ ). 
Physical activity, stress, and recovery

Table 1. Characteristics of the study population at baseline, 10-week post-intervention, and 36-week follow-up

\begin{tabular}{|c|c|c|c|c|c|c|c|c|c|c|c|c|}
\hline & \multicolumn{4}{|l|}{ All } & \multicolumn{4}{|c|}{ Female } & \multicolumn{4}{|c|}{ Male } \\
\hline & $N$ & Mean & SD & Range & $N$ & Mean & SD & Range & $N$ & Mean & SD & Range \\
\hline Age & 221 & 47.8 & 7.7 & $26-60$ & 185 & 48.3 & 7.5 & $26-60$ & 36 & 45.4 & 8.7 & $31-60$ \\
\hline Height & \multicolumn{9}{|c|}{ Body weight } & 179.7 & 6.7 & 167.5-195.6 \\
\hline Baseline & 221 & 87.7 & 11.6 & $64.0-120.1$ & 185 & 85.4 & 10.5 & $64.0-113.9$ & 36 & 99.6 & 9.8 & $83.8-120.1$ \\
\hline 10 weeks & 208 & 87.5 & 11.8 & 64.9-118.8 & 173 & 85.0 & 10.7 & $64.9-116.2$ & 35 & 99.5 & 9.8 & $83.8-118.8$ \\
\hline 36 weeks & 199 & 86.6 & 12.1 & $62.4-123.1$ & 165 & 84.2 & 11.1 & $62.4-123.1$ & 34 & 98.4 & 9.9 & $78.4-119.4$ \\
\hline \multicolumn{13}{|l|}{ Body fat $\%$} \\
\hline Baseline & 221 & 38.6 & 6.6 & $12.8-50.8$ & 185 & 40.7 & 4.6 & $28.4-50.8$ & 36 & 28.0 & 5.4 & $12.8-35.1$ \\
\hline 10 weeks & 207 & 38.5 & 6.8 & $14.7-50.4$ & 172 & 40.7 & 4.7 & $26.2-50.4$ & 35 & 27.8 & 5.2 & $14.7-35.0$ \\
\hline 36 weeks & 199 & 37.8 & 7.0 & $14.5-51.5$ & 165 & 39.9 & 5.0 & $26.0-51.5$ & 34 & 27.2 & 5.6 & $14.5-36.4$ \\
\hline \multicolumn{13}{|c|}{ Body mass index } \\
\hline Baseline & 221 & 31.1 & 3.1 & $25.3-40.1$ & 185 & 31.2 & 3.2 & $25.3-40.1$ & 36 & 30.8 & 2.6 & $26.3-37.0$ \\
\hline 10 weeks & 208 & 31.1 & 3.2 & $25.0-40.3$ & 173 & 31.2 & 3.3 & $25.0-40.3$ & 35 & 30.8 & 2.7 & $26.0-36.6$ \\
\hline 36 weeks & 199 & 30.7 & 3.3 & $24.0-40.6$ & 165 & 30.8 & 3.4 & $24.0-40.6$ & 34 & 30.5 & 2.9 & $26.1-36.0$ \\
\hline \multicolumn{13}{|c|}{ Physical activity (MET index*) } \\
\hline Baseline & 221 & 3.2 & 3.2 & $0.01-18.0$ & 185 & 3.0 & 2.9 & $0.01-15.3$ & 36 & 4.3 & 4.2 & $0.1-18.0$ \\
\hline 10 weeks & 207 & 3.2 & 2.8 & $0.01-13.8$ & 173 & 3.0 & 2.6 & $0.01-13.8$ & 34 & 3.9 & 3.6 & $0.1-12.8$ \\
\hline 36 weeks & 194 & 3.4 & 3.1 & $0.01-15.3$ & 163 & 3.3 & 3.0 & $0.01-15.3$ & 31 & 3.9 & 3.4 & $0.2-13.8$ \\
\hline \multicolumn{13}{|c|}{ Perceived Stress Scale sum score } \\
\hline Baseline & 221 & 26.5 & 7.6 & $7-52$ & 185 & 26.5 & 7.9 & $7-52$ & 36 & 26.2 & 5.8 & $17-38$ \\
\hline 10 weeks & 206 & 23.9 & 8.3 & $6-54$ & 173 & 24.3 & 8.4 & $6-54$ & 33 & 22.1 & 7.3 & $10-39$ \\
\hline 36 weeks & 196 & 21.9 & 8.7 & $2-50$ & 165 & 22.1 & 9.0 & $2-50$ & 31 & 21.3 & 6.6 & $10-45$ \\
\hline \multicolumn{13}{|c|}{ Stress index (24 h) } \\
\hline Baseline & 221 & 163.0 & 48.2 & $88.5-455.8$ & 185 & 163.2 & 48.4 & $88.5-455.8$ & 36 & 162.2 & 47.8 & $89.1-308.2$ \\
\hline 10 weeks & 174 & 157.7 & 43.9 & $84.0-445.7$ & 148 & 158.5 & 44.3 & $84.0-445.7$ & 26 & 153.5 & 42.0 & $99.0-274.6$ \\
\hline 36 weeks & 172 & 161.3 & 47.0 & $82.2-347.3$ & 142 & 160.6 & 46.1 & $82.2-347.3$ & 30 & 164.8 & 52.0 & $94.3-280.7$ \\
\hline \multicolumn{13}{|c|}{ Recovery index (sleep) } \\
\hline Baseline & 221 & 71.9 & 31.9 & $7.6-201.1$ & 185 & 70.9 & 31.9 & 7.6-201.1 & 36 & 77.0 & 32.2 & $23.2-147.1$ \\
\hline 10 weeks & 174 & 71.9 & 30.3 & $13.7-194.9$ & 149 & 70.8 & 30.1 & $13.7-194.9$ & 26 & 78.2 & 31.3 & $32.8-153.4$ \\
\hline 36 weeks & 171 & 75.4 & 33.5 & $8.0-189.0$ & 141 & 73.9 & 34.1 & $8.0-189.0$ & 30 & 82.5 & 30.4 & $26.2-156.1$ \\
\hline
\end{tabular}

The Wald test showed statistically significant differences in the mean values between the time periods in PSS sum score, body weight, body fat percentage, and BMI. There were no statistically significant differences in the mean values between the time periods in MET index, stress index, and recovery index.

*MET-h/day, based on a physical activity questionnaire.

The HRV data included in the analysis of this study consisted of successfully recorded work days, with an allowed maximum of $15 \%$ regarding the percentage of detected and corrected artifacts in R-R intervals. If participant's measurement period included two or more work/days, an average was calculated and the mean values of the stress and recovery indices were used in the analysis. At the baseline, 187 participants had HRV data from two consecutive work days and the Pearson correlation coefficient for these days was 0.90 for stress index and 0.77 for recovery index. For each monitored day, the participants reported their alcohol consumption in standard units of approximately $12 \mathrm{~g}$ of ethanol (one unit: 33 centiliters [cL] beer, $12 \mathrm{cL}$ red or white wine, $8 \mathrm{cL}$ fortified wine, or $4 \mathrm{cL}$ liquor), working hours, and sleeping hours in measurement diaries.

Subjective stress was assessed using the 14-item PSS (Cohen et al., 1983), which measures the degree to which situations in one's life during the preceding month have been stressful on a 5 -point scale ranging from 0 (never) to 4 (very often). The PSS sum score is calculated by reversing the scores of the seven positive items and then summarizing the scores of all 14 items. Internal reliability (Cronbach's $\alpha$ ) was 0.86 for baseline PSS, 0.90 for 10 weeks, and 0.92 for 36 weeks.

PA was assessed using a validated questionnaire with questions including present activity and changes in PA within the past 2 months. These questions covered both leisure-time PA and commuting activity and were related to the intensity, duration, and frequency of PA. To describe the intensity of the form of the activity, a multiple of the resting metabolic rate (MET) was assigned for each activity. The MET index for each form of PA was calculated by multiplying the intensity (MET), duration (h), and frequency of the activity. The MET index was expressed as the sum score of different activities (MET-h/d) (Kujala et al., 1998; Waller et al., 2007).

The assessment of body composition included measurement of the whole body fat percentage using bioelectrical impedance analysis (InBody720; Kuopio/Tanita BC-418 MA, Jyväskylä, Helsinki) in the morning after $10-12 \mathrm{~h}$ of fasting. Body weight and height were also measured during the same laboratory visit.

\section{Statistical analysis}

Our aim was to investigate the association between PA and objective HRV-based stress and recovery with subjective stress during a 9-month study period. Wald test was used to analyze the differences in means between the time points. Because the study design included repeated measures over time, we used latent growth curve modeling (LGM) (Bollen \& Curran, 2006). To answer our research question, we used LGM in the structural equation framework. In LGM, the initial level and the change of each construct across the three measurement points were modeled as separate latent factors (Figs 1 and 2). 
Föhr et al.

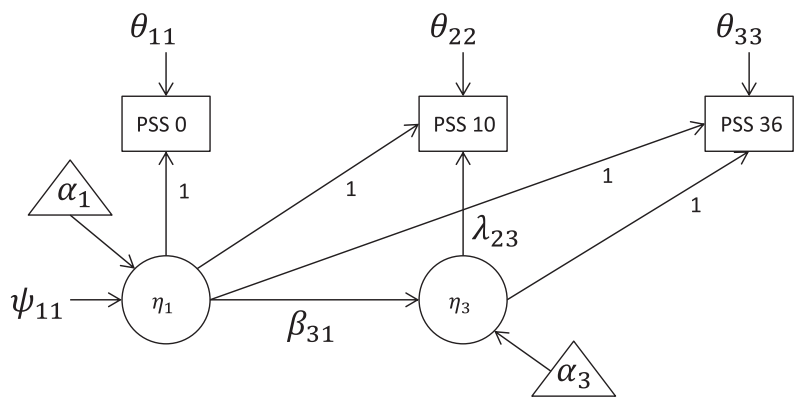

Fig. 1. A latent growth model for investigating the change in subjective stress at the three measurement points (Perceived Stress Scale [PSS] 0, PSS 10, and PSS 36). Residual variances $\left(\theta_{11}, \theta_{22}, \theta_{33}\right)$, estimated mean $\left(\alpha_{1}\right)$, variance $\left(\psi_{11}\right)$, latent level factor $\left(\eta_{1}\right)$, latent slope factor $\left(\eta_{3}\right)$, regression coefficient $\left(\beta_{31}\right)$, factor loading $\left(\lambda_{23}\right)$, and intercept $\left(\alpha_{3}\right)$.

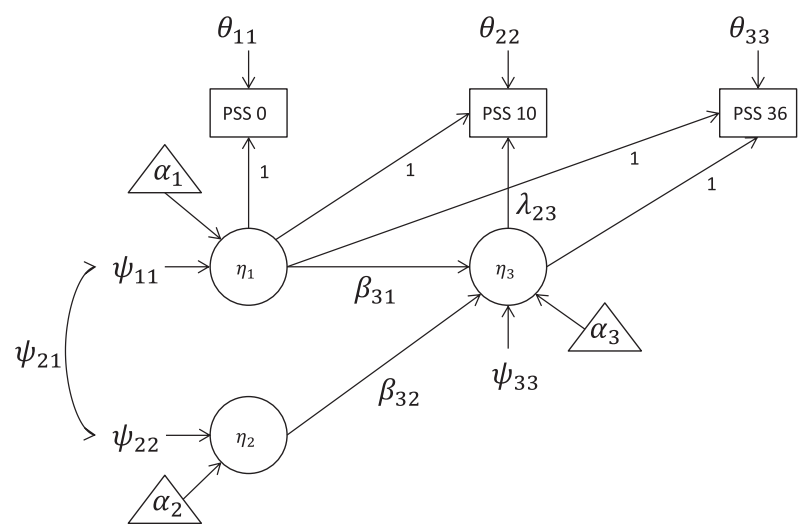

Fig. 2. A latent growth model for investigating the effect of the initial level of the predictor variables on the change in subjective stress at the three measurement points (Perceived Stress Scale [PSS] 0, PSS 10, PSS 36). Residual variances $\left(\theta_{11}, \theta_{22}, \theta_{33}\right)$, estimated means $\left(\alpha_{1}, \alpha_{2}\right)$, variances $\left(\psi_{11}, \psi_{22}\right)$, residual variance $\left(\psi_{33}\right)$, residual covariance $\left(\psi_{21}\right)$, latent level factors $\left(\eta_{1}\right)$, latent slope factor $\left(\eta_{3}\right)$, regression coefficients $\left(\beta_{31}, \beta_{32}\right)$, factor loading $\left(\lambda_{23}\right)$, and intercept $\left(\alpha_{3}\right)$.

By setting the first measurement point as the reference point, the latent level factor describes the mean level of the construct at baseline and its individual variation (e.g., latent level factor predicts the mean level of baseline and individual variation in baseline, the predictions of which are also equal to the second and third measurement points because the first measurement is set as the reference point). The latent slope factor, in turn, describes the mean change in each construct from the first to the last measurement point and individual variation in this change. The change used here refers to the non-linear change rate, which is affected by estimated factor loading of the second measurement point. An important element of LGM is its ability to deal with both the mean change rate and individual deviation from the mean change rate over time. This modeling feature enables an estimate of the associations of growth components for two parallel LGMs.

The LGM specifications for PSS over time are presented in Fig. 1. In the model, the factor loadings of the change were set to zero at first (PSS 0), set to one at third (PSS 36), and allowed to be freely estimated at the second measurement (PSS 10). Furthermore, all intercepts of the observed variables were set to zero and the factor means/intercepts were freely estimated. Using this specification, the parallel LGM for PSS and one predictor variable (MET index, stress index, recovery index) at a time was estimated. In addition, we included gender, age, medication use, alcohol consumption, and intervention group one at a time as covariates into the models.

The Mplus program (version 7.1) was used in the analysis of this study. The significance level of the study was set at 0.05 . All the models were estimated using the full information maximum likelihood method with the standard errors and chi-square test, which are robust in the case of non-normal distribution (MLR estimator in Mplus). The missing values were assumed to be missing at random (Muthén \& Muthén, 1998-2012). In addition, the model fit was evaluated using the chi-square $\left(\chi^{2}\right)$ test, root mean square error of approximation (RMSEA), comparative fit index (CFI), Tucker Lewis index (TLI), and standardized root mean square residual (SRMR). Non-significant $\chi^{2}$ test, RMSEA lower than 0.06, CFI and TLI greater than 0.95 , and SRMR lower than 0.08 indicated a good-fitting model (Hu \& Bentler, 1999).

\section{Results}

The Wald test showed statistically significant differences in the mean values between the time periods in PSS sum score, body weight, body fat percentage, and BMI. There were no statistically significant differences in the mean values between the time periods in MET index, stress index, and recovery index (Table 1).

There was significant individual variation in the latent level and slope factors, and decline in the average PSS sum score, but the average and individual levels of stress index, recovery index, and MET index were rather stable during the 9-month study period. Due to the non-significant average growth and variance in growth of these variables across the three measurement points, only the latent level factor is included in the parallel LGM (Fig. 2). The results of the model fit evaluation tests for the parallel LGMs are presented in Table 2. For the LGM of the PSS alone (Fig. 1), the model was saturated (e.g., the model fit was perfect).

The initial level of PSS was significantly associated with the change in PSS (standardized $\beta=-0.536$, $P<0.001$ ). Higher initial level of PSS predicted a larger decline in PSS. The initial levels of the MET index (standardized $\beta=-0.266, P<0.001$ ), stress index (standardized $\beta=0.259, P=0.001$ ) and recovery index (standardized $\beta=-0.250, P<0.01$ ) were

Table 2. The results of the model fit evaluation tests for the parallel growth models; chi-square $\left(\chi^{2}\right)$ test, root mean square error of approximation (RMSEA), comparative fit index (CFI), Tucker Lewis index (TLI), and standardized root mean square residual (SRMR)

\begin{tabular}{lcrlll}
\hline & $\chi^{2}(P)$ & RMSEA & CFI & TLI & SRMR \\
\hline Model 1 & 0.271 & 0.031 & 0.994 & 0.991 & 0.029 \\
Model 2 & 0.571 & $<0.001$ & 1.000 & 1.000 & 0.029 \\
Model 3 & 0.524 & $<0.001$ & 1.000 & 1.000 & 0.029 \\
\hline
\end{tabular}

Model 1: Perceived Stress Scale (PSS) and MET index; Model 2: PSS and stress index; Model 3: PSS and recovery index. 
significantly associated with the change in PSS. The non-standardized values for the parallel LGMs are presented in Table 3. Figs 3-5 illustrate the predicted changes in PSS that were influenced by the initial MET index, stress index, and recovery index levels. Higher initial level (average +1 standard deviation) of the stress index predicted a weaker decline in PSS (Fig. 3), whereas higher initial levels of the recovery (Fig. 4) and MET indices (Fig. 5) predicted a larger decline in PSS during the study period.

Neither body fat percentage nor BMI was associated with changes in PSS. Adjustment for age, sex, current medication or changes in medication during the study period, alcohol consumption, or intervention group did not affect the results. Age and current medication were significantly associated with the initial level of the stress index and recovery index; however, age and medication were not associated with changes in PSS over time.

\section{Discussion}

This study investigated the association between PA and HRV-based stress and recovery with subjective stress among overweight and psychologically distressed working-age adults over a 9-month study period. Interestingly, we found that the initial levels of PA and objective stress and recovery were associated with an overall change in subjective stress. The results suggest that individuals who feel stressed but have lower objective stress, have higher objective recovery, or are more physically active than average are prone to larger declines in subjective stress in the long term. This study utilized novel technology, and the results are in line with previous studies and traditional HRV measurement methods. Furthermore, our results provide additional information about the health benefits of PA.

We found that initially higher level of PA was associated with a larger decline in subjective stress. This suggests that PA has beneficial effects on the individual's ability to decrease the level of subjective stress. To clarify, individuals who experience stress and at the same time are physically active benefit from their PA. They are more prone to lower the level of subjective stress compared with those who are inactive and psychologically stressed. In line with this study, a meta-analysis of work place PA interventions suggests that an increase in PA may decrease job stress among other health benefits (Conn et al., 2009). From the perspective of physiological stress, PA may increase the ability of the cardiovascular system to control responses to acute stressors and speed up cardiovascular recovery from stress (Forcier et al., 2006). Our finding showing the association between higher initial PA level and a larger decline in subjective stress provides additional

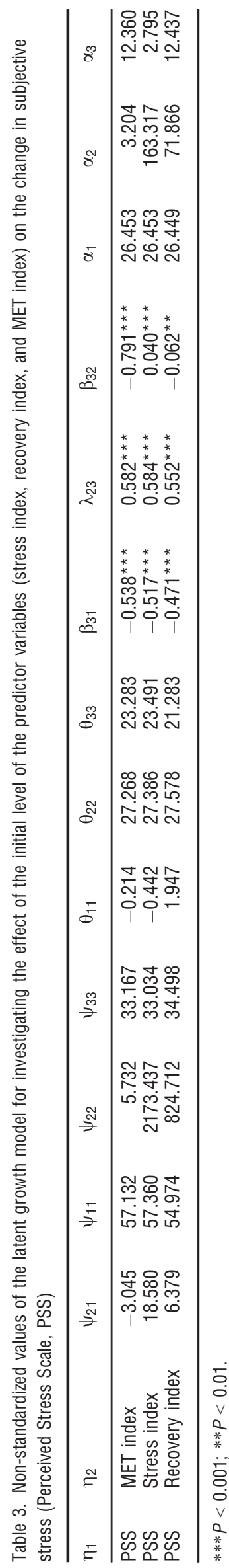


Föhr et al.

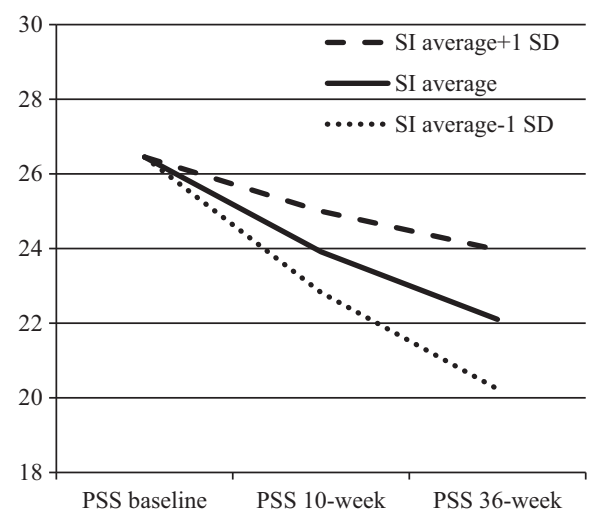

Fig. 3. The effect of the initial level of the stress index (SI); average, higher than average (average +1 standard deviation [SD]), and lower than average (average $-1 \mathrm{SD}$ ) SI on the change in subjective stress (PSS) during a 9-month study period.

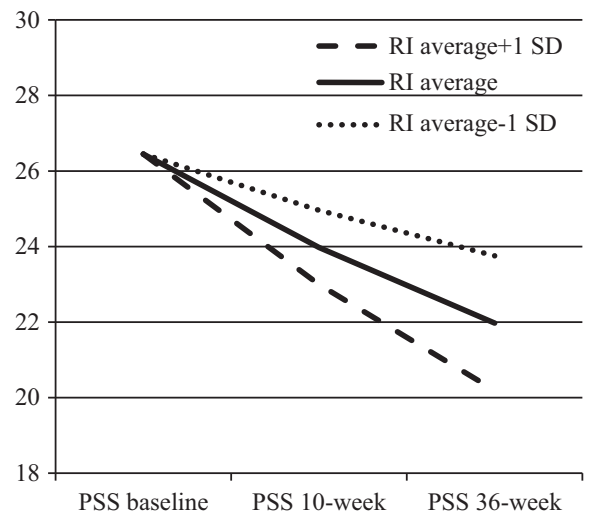

Fig. 4. The effect of the initial level of the recovery index (RI); average, higher than average (average +1 standard deviation [SD]), and lower than average (average -1 SD) RI on the change in subjective stress (PSS) during a 9-month study period.



Fig. 5. The effect of the initial level of the MET index (METI); average, higher than average (average +1 standard deviation [SD]), and lower than average (average $-1 \mathrm{SD}$ ) METI on the change in subjective stress during a 9-month study period. evidence about the health benefits of PA. Based on our results, we are not able to draw any conclusions about the effect of increased PA on subjective or objective stress because there was no significant change in PA among our participants. It is suggested that the greatest improvements in health status are seen when people who are least fit become physically active and people who engage in exercise at levels above the general PA recommendations are likely to gain further health benefits (Warburton et al., 2006). More research about the relationships between increased PA, HRV profiles, and subjective stress is needed. However, it is challenging to increase people's PA, and worksite PA interventions are thought to be associated with relatively small increases in PA (Bravata et al., 2007).

Previous studies using HRV measures have focused mainly on cross-sectional associations of subjective stress with objective stress. Subjective stress is found to be associated with decreased HRV (Jarczok et al., 2013). For instance, Uusitalo et al. (2011) found that lower HRV was associated with higher feelings of stress during work days. They found that daytime irritation had a negative effect on HRV the following night. In line with this, Clays et al. (2011) found self-reported work stress to be associated with high HRV-based objective stress among 653 male workers. Additionally, there is some evidence on the association between HRV-based objective stress and subjective stress in longitudinal settings, but the results are somewhat inconsistent. In the study by Pärkkä et al. (2009), an intervention including lectures about stress management and exercise had a positive effect on participants' subjective stress levels. Interestingly, the authors found that the amount of both objective stress and recovery increased due to high subjective stress on a stressful day during the intervention. However, the study did not report significant changes in objective stress or recovery between pre- and post-intervention. According to Pärkkä et al. (2009), the observed increase in recovery on a stressful day may be caused by inactivity on that day. This is a reasonable conclusion because of the lack of the time determined as PA so that there is more time for other states of the body such as stress and recovery. Melville et al. (2012) found that $15 \mathrm{~min}$ of yoga and meditation were associated with reduced subjective stress and positive changes in HRV-based indicators of objective stress. The change in subjective stress was maintained 15-min post-intervention. Cheema et al. (2013) found that the positive change in participants' self-reported anxiety followed by a 10-week yoga intervention did not result in improvements in their HRV profiles. To conclude, the results of the previous studies are in line with the present study suggesting that positive changes in individuals' sub- 
jective experience of stress may be associated with short-time positive changes in their HRV profile. However, these results highlight that objective and subjective stress react differently so that HRV seems to be more stable than subjective experience of stress in long term. It is well demonstrated that after transient perturbations, for instance induced by mild exercise or short-acting vasodilators, short-term measures of HRV rapidly return to baseline. This return may be prolonged after more powerful stimuli, such as maximal exercise or long-acting drugs (Task Force, 1996). However, more research is needed on how to achieve more permanent changes in HRV profiles.

HRV has been proposed to be a good indicator for investigating the physiological effects of stress. It is even suggested that the association between stress and HRV is more obvious than the association of plasma catecholamines or cortisol secretion with stress (Chandola et al., 2010). There is a recognized need for further research about the validity and reliability of HRV measurement. Among other differences between the methods of the existing HRV studies, the length of the HRV recordings varies greatly. Only short-term recordings of a few minutes in laboratory settings are used in some studies, whereas other studies utilize real-life recordings from $24 \mathrm{~h}$ to over several days. The results of Cipryan and Litschmannova (2013) indicate that researchers should be very cautious when drawing conclusions based on short-term HRV analysis. As HRV measurement is dependent on the duration of the R-R interval recording (Cipryan \& Litschmannova, 2013), the development of long-term methods is necessary to improve the reliability of HRV analysis. Stability of HRV measures derived from 24-h recording is suggested to be high (Task Force, 1996). In this study, the reliability of the HRV-based measurement of stress and recovery between two consecutive days at baseline was quantified and the correlation for both stress index and recovery index was high. The novel method used in this study has been utilized in previous studies (e.g., Uusitalo et al., 2011; Teisala et al., 2014) and the findings of these studies further support the validity and reliability of this HRV-based method. The method has been validated against neuroendocrine responses to stress, and the indicators of stress and recovery during sleep have been found to be associated with free salivary cortisol response after awakening (Rusko et al., 2006).

It is a strength of the present study that it utilized novel technology and that the HRV data were recorded in real-life settings over a rather long-time period, usually two work days. Hence, the validity and reliability of the present results can be considered to be strengthened due to the long-term HRV-based method used. The validity and reliability of the results was further enhanced by the fact that if the 24-h recording was unsuccessful (over $15 \%$ regarding the percentage of detected and corrected artifacts in R-R intervals), the day was excluded from the analysis. Traditional measures of HRV are sensitive to several confounding factors; thus, the average value of a traditional HRV measure from the measurement day is not very informative. The method presently used produced stress- and recovery-related variables based on the HR, HRV, and respiratory variables. First, the method recognizes the different physiological states and provides information about the magnitude of the physiological reactions. These variables can be considered as informative to both health-care professionals and patients/clients. It is difficult to compare the present real-life HRV-based findings with the previous ones obtained from short-term HRV recordings and those that used different measures than applied in this study. However, it is important to develop HRV measurement and to generate methods that provide informative and suitable variables that can be applied for general health care and lifestyle counseling.

As mentioned above, confounding factors that affect HRV are encountered when using traditional time-domain or frequency-domain measures of HRV. For instance, age, diseases like hypertension and diabetes, ethnicity, medication, smoking, alcohol and caffeine consumption, level of PA, and breathing are potential confounders in HRV studies (Task Force, 1996; Jarczok et al., 2013). Additionally, the history of individual's medication may be a potential confounder. It is suggested that antidepressants cause a decrease in cardiac vagal control that is only partly reversible after discontinuing the medication (Licht et al., 2010). Previous studies with traditional HRV variables often do adjust for at least age, but there is a great variation in the observation of the other potential confounders (Jarczok et al., 2013). We investigated the effect of gender, age, alcohol consumption, and intervention group on the associations found. Although, this study excluded subjects who used $\alpha$ - or $\beta$-blockers, our participants included individuals using regular medication such as other cardiac medications or psychopharmacological, metabolic, or analgesic medication. Therefore, we also accounted for current regular medication and changes in medication during the study period in our present analysis. These adjustments did not affect the present results. However, we did not have the information about participants' long-term history of medications that may have effect on HRV (Licht et al., 2010). This study did not investigate differences between the three intervention groups and the control group. We were interested in the associations between PA and objective stress and recovery with subjective stress independent of the intervention. 


\section{Föhr et al.}

Self-report measures are influenced by several factors, and there is a risk for measurement bias. In stress assessment, however, subjective assessment cannot be totally replaced by objective methods. Although selfreports always include the risk of subjective biases, replacing self-reports with stress-related physiological measurements is not recommended. In stress assessment, it would be optimal to complement self-report measures with other objective measures (Semmer et al., 2003). For example, our results suggest that using both objective and subjective stress assessment methods can predict more detailed information about individuals' stress and changes in their subjective stress. Additionally, attrition is an issue in longitudinal studies and needs to be considered in the analysis. In our analysis, the missing values were assumed to be missing at random, and all the available data and possible differences between dropouts and completers were considered in the analyses. The dropouts did not differ from the completers at baseline according to PSS sum score, stress index, recovery index, or MET index. The dropout rate was slightly greater among women.

This study is unique in its focus on the association between the initial levels of PA and HRV-based indicators of stress and recovery with changes in subjective stress in a longitudinal setting. HRV recording in real life involves several confounding factors, and there is a need for the development of HRV methods. This study utilized novel HRV-based method which acknowledges the individuality of HRV. First, the present results highlight the importance of PA in stress management. Second, the findings suggest that it is important to acknowledge both the subjective and the objective aspects of stress in stress assessment and management. The present results indicate that factors such as PA and objective stress and recovery affect the individual's perception of subjective stress. Further the results highlight the difference between objective and subjective stress as the positive change in subjective stress was not observed in objective indices of stress or recovery. Hence, the results give reason for assuming that positive changes in HRV-based objective stress and recovery require additional changes in lifestyle, such as increase in PA. Nevertheless, more research about the relationship between subjective and objective stress is needed because of the limited evidence about the association between changes in physiological and psychological stress variables in longitudinal settings. Further- more, intervention studies about the association of increased PA with both subjective and objective stress are warranted. This study suggests that the novel HRV-based technology used in the assessment of objective stress and recovery in this study can provide important information about individual's stress and recovery. These kinds of methods for monitoring stress in real life could help to obtain an early identification of stress and may alert the individual to engage in corrective actions, such as PA.

\section{Perspective}

Sedentary lifestyle and stress are major concerns in today's society because they are risk factors for multiple adverse health outcomes. HRV may be a feasible method for stress assessment. However, it is important to develop HRV-based long-term methods that account for the individuality of HRV. This longitudinal study utilized novel HRV-based technology and the results suggest that high PA and objectively assessed low stress and good recovery have positive effects on changes in subjective stress in the long term. These results provide important information about the efforts needed for stress management to improve individual's health and wellness. This information is valuable for health policy, and the results are useful for health-care professionals in their daily clinical work.

Key words: MET index, physiological stress, psychological stress, stress assessment, stress management.

\section{Acknowledgements}

This study was supported by the SalWe Research Program for Mind and Body (Tekes - the Finnish Funding Agency for Technology and Innovation, grant 1104/10). T. Föhr's work was supported by a personal grant from the Juho Vainio Foundation.

\section{Conflict of interest}

T. Myllymäki is an employee of and H. Rusko is a stockowner in Firstbeat Technologies Ltd. They did not contribute to writing the conclusions of the study. The other authors declare that they have no conflict of interests.

\section{References}

Bollen KA, Curran PJ. Latent curve models: a structural equation perspective. Hoboken, NJ: John Wiley \& Sons, 2006.
Bravata DM, Smith-Spangler C, Sundaram V, Gienger AL, Lin N, Lewis R, Stave CD, Olkin I, Sirard JR. Using pedometers to increase physical activity and improve health: a systematic review. JAMA 2007: 298: 2296-2304. 
Chandola T, Heraclides A, Kumari M. Psychophysiological biomarkers of workplace stressors. Neurosci Biobehav Rev 2010: 35: 51-57.

Cheema BS, Houridis A, Busch L, Raschke-Cheema V, Melville GW, Marshall PW, Chang D, Machliss B, Lonsdale C, Bowman J, Colagiuri B. Effect of an office worksite-based yoga program on heart rate variability: outcomes of a randomized controlled trial. BMC Complement Altern Med 2013: 13: 82: doi:10.1186/1472-6882.

Cipryan L, Litschmannova M. Intraday and inter-day reliability of heart rate variability measurement. J Sports Sci 2013: 31: 150-158.

Clays E, De Bacquer D, Crasset V, Kittel F, De Smet P, Kornitzer M, Karasek R, De Backer G. The perception of work stressors is related to reduced parasympathetic activity. Int Arch Occup Environ Health 2011: 84: 185-191.

Cohen S, Kamarck T, Mermelstein R. A global measure of perceived stress. J Health Soc Behav 1983: 24: 385-396.

Conn VS, Hafdahl AR, Cooper PS, Brown LM, Lusk SL. Meta-analysis of workplace physical activity interventions. Am J Prev Med 2009: 37: 330-339.

Dodge R, Daly AP, Huyton J, Sanders LD. The challenge of defining wellbeing. Int J Wellbeing 2012: 2: 222-235: doi:10.5502/ijw.v2i3.4.

Firstbeat Technologies Ltd (2014) White papers. Available at http:// www.firstbeat.com/physiology/whitepapers (accessed May 15, 2015).

Forcier K, Stroud LR, Papandonatos GD, Hitsman B, Reiches M, Krishnamoorthy J, Niaura R. Links between physical fitness and cardiovascular reactivity and recovery to psychological stressors: a meta-analysis. Health Psychol 2006: 25: 723-739.

Gerber M, Pühse U. Review article: do exercise and fitness protect against stress-induced health complaints? A review of the literature. Scand $\mathbf{J}$ Public Health 2009: 37: 801-819.

$\mathrm{Hu}$ L, Bentler PM. Cutoff criteria for fit indexes in covariance structure analysis: conventional criteria versus new alternatives. Struct Equ Model 1999: 6: 1-55.

Jarczok MN, Jarczok M, Mauss D, Koenig J, Li J, Herr RM, Thayer JF. Autonomic nervous system activity and workplace stressors - a systematic review. Neurosci Biobehav Rev 2013: 37: 1810-1823.

Kettunen J, Saalasti S, inventors; Firstbeat Technologies Ltd, assignee:
Procedure for deriving reliable information on respiratory activity from heart period measurement. United States patent US 20050209521A1; 2005.

Kettunen J, Saalasti S, inventors; Firstbeat Technologies Ltd, assignee: Procedure for detection of stress by segmentation and analyzing heart beat signal. United States patent US 007330752B2; 2008.

Kinnunen M. Allostatic load in relation to psychosocial stressors and health. PhD Thesis. University of Jyväskylä, Finland, 2005.

Kujala UM. Evidence on the effects of exercise therapy in the treatment of chronic disease. Br J Sports Med 2009: 43: 550-555.

Kujala UM, Kaprio J, Sarna S, Koskenvuo M. Relationship of leisure-time physical activity and mortality: The Finnish Twin Cohort. JAMA 1998: 279: 440-444.

Lappalainen R, Pulkkinen P, van Gils M, Pärkkä J, Korhonen I. Longterm self-monitoring of weight: a case study. Cogn Behav Ther 2005: 34: 108-114.

Lappalainen R, Sairanen E, Järvelä E, Rantala S, Korpela R, Puttonen S, Kujala UM, Myllymäki T, Peuhkuri K, Mattila E. The effectiveness and applicability of different lifestyle interventions for enhancing wellbeing: the study design for a randomized controlled trial for persons with metabolic syndrome risk factors and psychological distress. BMC Public Health 2014: 14: 310. doi:10.1186/1471-2458-14310.

Licht CM, de Geus EJ, van Dyck R, Penninx BW. Longitudinal evidence for unfavorable effects of antidepressants on heart rate variability. Biol Psychiatry 2010: 68: 861-868: doi:10.1016/ j.biopsych.2010.06.032.

Makowska Z, Merecz D, Moscicka A, Kolasa W. The validity of general health questionnaires, GHQ-12 and GHQ-28, in mental health studies of working people. Int $\mathbf{J}$ Occup Med Environ Health 2002: 15: 353-362.

Martinmäki K, Rusko H, Kooistra L, Kettunen J, Saalasti S.

Intraindividual validation of heart rate variability indexes to measure vagal effects on hearts. Am J Physiol Heart Circ Physiol 2006: 290: 640-647.

Melville GW, Chang D, Colagiuri B, Marshall PW, Cheema BS. Fifteen minutes of chair-based yoga postures or guided meditation performed in the office can elicit a relaxation response. Evid Based Complement
Alternat Med 2012: 2012: 501986. doi:10.1155/2012/501986.

Muthén LK, Muthén BO. Mplus User's Guide. 7th edn. Los Angeles, CA: Muthén \& Muthén, 1998-2012.

Pärkkä J, Merilahti J, Mattila EM, Malm E, Antila K, Tuomisto MT, Saarinen AV, van Gils M, Korhonen I. Relationship of psychological and physiological variables in long-term self-monitored data during work ability rehabilitation program. IEEE Trans Inf Technol Biomed 2009: 13: 141-151.

Pedersen BK, Saltin B. Evidence for prescribing exercise as therapy in chronic disease. Scand J Med Sci Sports 2006: 16: 3-63.

Physical Activity Guidelines Advisory Committee. Physical Activity Guidelines Advisory Committee Report, 2008. Washington, DC: US Department of Health and Human Services, 2008.

Porges SW. Vagal tone: a physiologic marker of stress vulnerability. Pediatrics 1992: 90: 498-504.

Porges SW. Cardiac vagal tone: a physiological index of stress. Neurosci Biobehav Rev 1995: 19: 225-233.

Rusko H, Rönkä T, Uusitalo A, Kinnunen U, Mauno S, Feldt T, Kinnunen M-L, Martinmäki K, Hirvonen A, Hyttinen S, Lindholm H. Stress and relaxation during sleep and awake time, and their associations with free salivary cortisol after awakening. Nordic Ergonomics Society Congress, 2006. Available at: http:// www.firstbeat.com/ userData/firstbeat/download/ rusko_et_al_nes_2006_congress.pdf (accessed May 15, 2015)

Saalasti S. Neural networks for heart rate time series analysis. $\mathrm{PhD}$ Thesis. University of Jyväskylä, Finland, 2003.

Semmer NK, Grebner S, Elfering A. Beyond self-report: using observational, physiological, and situation-based measures in research on occupational stress. Res Occup Stress Well-Being 2003: 3: 205-263.

Task Force. Task Force of the European Society of Cardiology and the North American Society of Pacing and Electrophysiology Heart Rate Variability. Standards of measurement, physiological interpretation, and clinical use. Eur Heart J 1996: 93: 1043-1065.

Teisala T, Mutikainen S, Tolvanen A, Rottensteiner M, Leskinen T, Kaprio J, Kolehmainen M, Rusko H, Kujala UM. Associations of physical activity, fitness, and body 


\section{Föhr et al.}

composition with heart rate variability-based indicators of stress and recovery on workdays: a crosssectional study. J Occup Med Toxicol 2014: 9: 16: doi:10.1186/ 1745-6673-9-16.

Thayer JF, Yamamoto SS, Brosschot JF. The relationship of autonomic imbalance, heart rate variability and cardiovascular disease risk factors. Int J Cardiol 2010: 141: 122-131.

Thøgersen-Ntoumani C, Loughren EA, Kinnafick F, Taylor IM, Duda JL, Fox KR. Changes in work affect in response to lunchtime walking in previously physically inactive employees: a randomized trial. Scand J Med Sci Sports 2015: doi:10.1111/ sms.12398.
Uusitalo A, Mets T, Martinmäki K, Mauno S, Kinnunen U, Rusko H. Heart rate variability related to effort at work. Appl Ergon 2011: 42: 830-838.

Vanderlei LCM, Pastre CM, Hoshi RA, Carvalho TD, Godoy MF. Basic notions of heart rate variability and its clinical applicability. Rev Bras Cir Cardiovasc 2009: 24: 205 217.

Verberk WJ, Kroon AA, Lenders JW, Kessels AG, van Montfrans GA, Smit AJ, van der Kuy PH, Nelemans PJ, Rennenberg RJ, Grobbee DE, Beltman FW, Joore MA, Brunenberg DE, Dirksen C, Thien T, de Leeuw PW. Selfmeasurement of blood pressure at home reduces the need for antihypertensive drugs: a randomized controlled trial. Hypertens 2007: 50: 1019-1025.

Waller K, Kaprio J, Kujala U. Associations between long-term physical activity, waist circumference and weight gain: a 30-year longitudinal twin study. Int $\mathrm{J}$ Obes 2007: 32: 353-361.

Warburton DE, Nicol CW, Bredin SS. Health benefits of physical activity: the evidence. Can Med Assoc J 2006: 174: 801-880.

Zahorska-Markiewicz B, Kuagowska E, Kucio C, Klin M. Heart rate variability in obesity. Int $\mathrm{J}$ Obes Relat Metab Disord 1993: 17: 21-23. 